\title{
Penile plaque as predictor of an advanced anorectal carcinoma: A case report
}

\author{
Andrea Fabiani ${ }^{1}$, Alessandra Filosa ${ }^{2}$, Fabrizio Fioretti ${ }^{1}$, Gabriele Mammana ${ }^{1}$ \\ ${ }^{1}$ Unit of Urology, Surgical Department, ${ }^{2}$ Section of Pathological Anatomy, Department of Clinical Pathology, Macerata Hospital, \\ Area Vasta 3, ASUR Marche, Italy.
}

\begin{abstract}
Summary The secondary involvement of the penis by tumors from others organs is a rare event representing only $0.8 \%$ of overall metastasis in the genitourinary tract. The most frequent clinical findings is priapism, but occasionally, solitary metastases to the penile skin, mucosa of the glans, corpus spongiosum or lesions of the albuginea mimicking an induratio penis have been reported. We report a case of penile plaque predicting the relapse of an anorectal carcinoma. The precise etiology of this particular manifestation is not well understood and the prognosis is poor. There are no individual treatments with curative intent.
\end{abstract}

KeY WORDS: Penile induration; Metastasis; Penile neoplasms; Anorectal carcinoma.

Submitted 1 September 2014; Accepted 20 December 2014

\section{Case report}

A 78 year old man came to our attention presenting with a fast growing painless nodular lesion of the lateral surface of the root of the left corpus cavernosum of the penis. Medical history included an anorectal carcinoma for which the patient had undergone surgical resection and complementary radiation therapy 5 years before. Many years before, the patient underwent a surgical debridement of scrotal tissue due to a necrotizing fasciitis. Penile ultrasound evaluation revealed a nodular thickening of albuginea, inhomogeneous for echo pattern, containing hyperechoic spots with posterior shadowing and hypoechoic areas, sized $30 \mathrm{~mm} \times 27,5 \mathrm{~mm} \times$ $13,5 \mathrm{~mm}$, with minimal presence of color signal at ecocolor ultrasound assessment (Figures 1-2).

Excisional biopsy was performed with local anhestesia (penile ring block technique with a mixture of $5 \mathrm{ml}$ of $1 \%$ lidocaine and $5 \mathrm{ml}$ of plain $0.25 \%$ bupivacaine). Frozen section examination revealed a malignant lesion

Figure 2.

Longitudinal ultrasound image of the nodule. Cavernous tissue is infiltrated by the solid nodule. Tunica albuginea is non remarkable.

\section{Figure 1.}

Transversal ultrasound image of circumscribed nodule within the root of left corpus cavernosum showing an inhomogeneous echostructural pattern consisting in hyperechoic spots with posterior shadowing and hypoechoic areas with minimal presence of color signal at ecocolor ultrasound assessment. Tunica albuginea is interrupted.
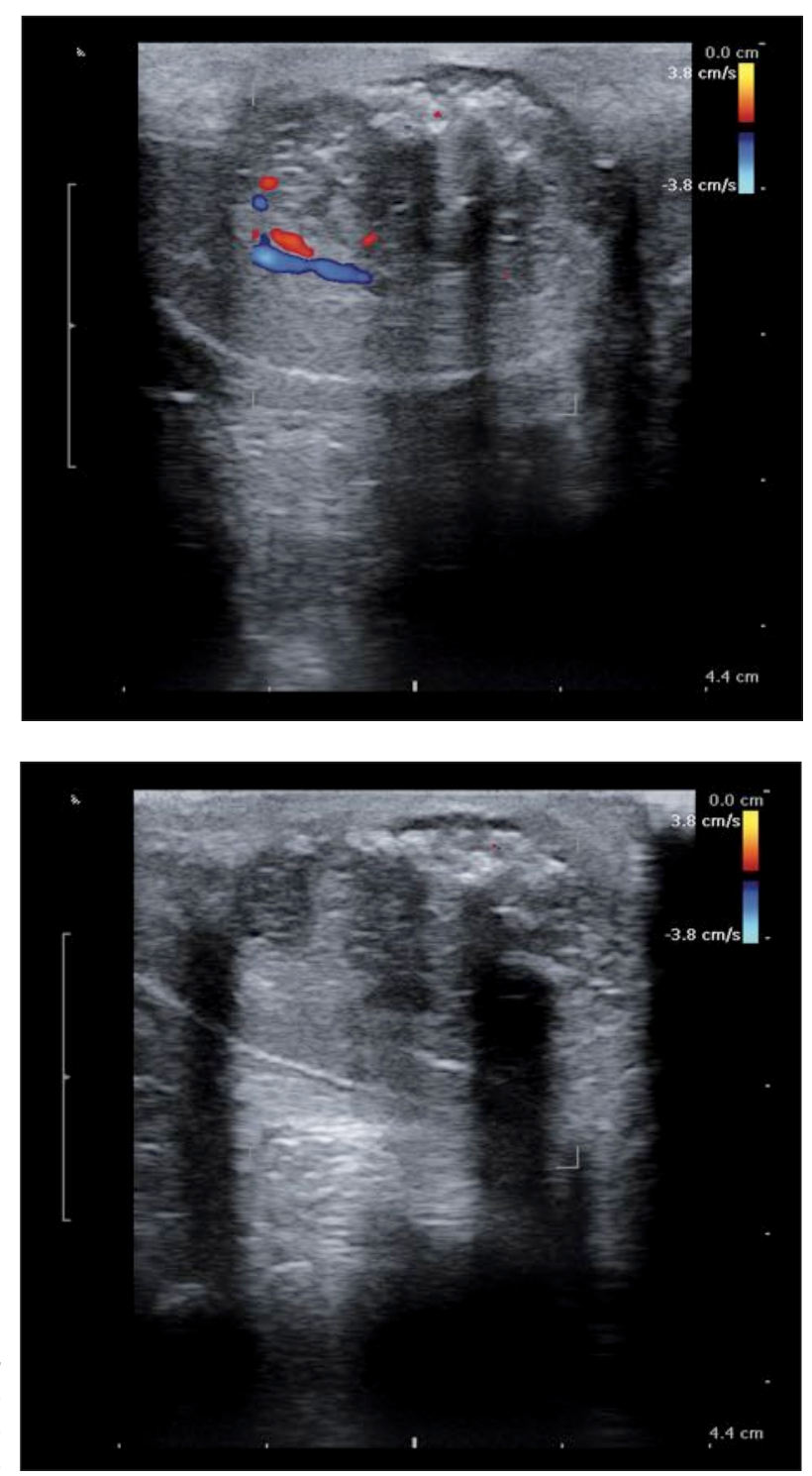


\section{Figure 3.}

Hystopathologic features of metastatic nodule excisional biopsy.

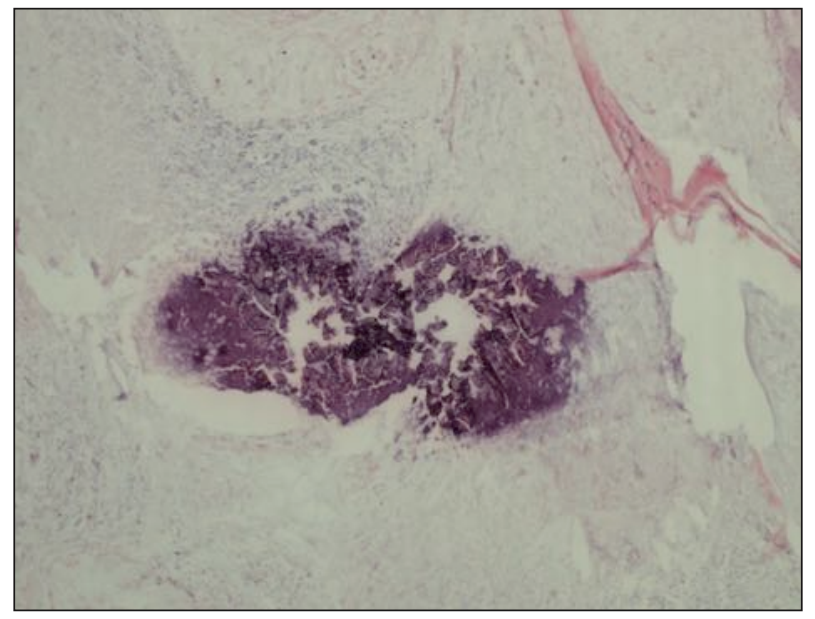

traceable to an adenocarcinoma (Figure 3). Patient was studied with a total body computed tomography (CT) scan and a recto-colonscopy with evidence of relapsed colorectal neoplasm. Two months later, the patient underwent a surgical ileostomy because of occlusion from rectal cancer recurrence. He died after 17 months after chemotherapy administration.

\section{Discussion}

Penis is rarely involved by metastatic spread of tumors from others organs. In a report of more than 600 tumors with metastases to the genitourinary tract, only $0.8 \%$ were located in this organ (1). The most frequent clinical findings is priapism but occasionally, solitary metastases to the penile skin, mucosa of the glans, corpus spongiosum or lesions of the albuginea mimicking an induratio penis have been reported. The urinary bladder and the prostate gland represent about the $70 \%$ of the primary tumors who give metastases to the penis (3). Most of the remaining metastases originate from tumors of the gastrointestinal tract, usually the rectosigmoid tract (2). In this case, the metastatic tumor was localized in the tunica albuginea in absence of documented widely dissemi- nated disease. At least prior to our clinic evaluation, the patient had no signs of recurrence of primitive disease. Only the examinations subsequently performed highlighted a recurrence of the anorectal carcinoma. Retrograde venous spread from the pudendal venous system appears to be the most probable way of spreading (2-3). Another mechanism may be a retrograde lymphatic dissemination into penile lymphatic channels after obstruction of inguinal and hypogastric nodes determined by anatomical disruption of lymphatic drainage after surgery or radiotherapy. The diagnosis could be difficult due to the non specific clinical and instrumental features. Only an histological evaluation of the biopsy could confirm the suspect. In cancer patients penile metastasis tends to be associated with a poor prognosis because its presence tends to be part of a widely disseminated disease.

The majority of patients dies within one year. There are no treatment of choice and the combination treatment with radiotherapy and chemotherapy in such advanced disease produces only occasional responses (2).

\section{Conclusion}

Despite the metastatic penile involvement can be considered a rare occurrence in visceral tumours, in cases of fast-growing penile induration with anamnesis of neoplasms and suspected ultrasonographic findings, an early histological assessment of penile lesion should be performed to avoid a misdiagnosis. Evaluation of progression of primitive tumor is mandatory because metastasis to the penis, in most cases, tends to be part of a widely disseminated disease.

\section{REFERENCES}

1. Bates AW, Baithun SI. Secondary tumours of the penis. J R Soc Med. 2002; 95:162-3.

2. Yuta Kimura, Dai Shida, Keiichi Nasu, et al. Metachronous penile metastasis from rectal cancer after total pelvic exenteration. World J Gastroenterol. 2012; 18:5476-5478;

3. Chaux A, Amin M, Cubilla AL, et al. Metastatic tumors to the penis: a report of 17 cases and review of the literature. Int J Surg Pathol. 2011; 19:597-606.

\section{Correspondence}

Andrea Fabiani, MD (Corresponding Author)

andreadoc1@libero.it

Fabrizio Fioretti, MD, PhD

fa.fioretti@libero.it

Gabriele Mammana, MD

gabriele.mammana@sanita.marche.it

Surgery Dpt, Section of Urology

ASUR Marche Area Vasta 3, Macerata Hospital, Italy

Alessandra Filosa, $M D, P h D$

alessandrafilosa@yahoo.it

Section of Pathological Anatomy, Department of Clinical Pathology,

Area Vasta 3, ASUR Marche, Macerata Hospital, Macerata, Italy 